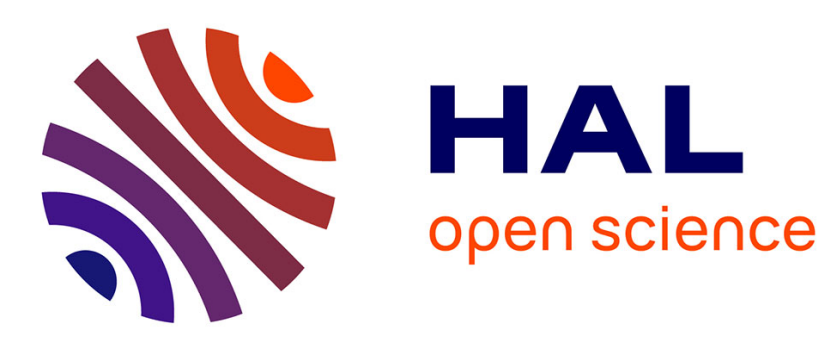

\title{
Système membrane - couche d'air mince chez transducteurs électrostatiques et piézoplastiques
}

Z. Skvor

\section{To cite this version:}

Z. Skvor. Système membrane - couche d'air mince chez transducteurs électrostatiques et piézoplastiques. Journal de Physique IV Proceedings, 1994, 04 (C5), pp.C5-3-C5-10. 10.1051/jp4:1994501. jpa-00252763

\section{HAL Id: jpa-00252763 https://hal.science/jpa-00252763}

Submitted on 1 Jan 1994

HAL is a multi-disciplinary open access archive for the deposit and dissemination of scientific research documents, whether they are published or not. The documents may come from teaching and research institutions in France or abroad, or from public or private research centers.
L'archive ouverte pluridisciplinaire HAL, est destinée au dépôt et à la diffusion de documents scientifiques de niveau recherche, publiés ou non, émanant des établissements d'enseignement et de recherche français ou étrangers, des laboratoires publics ou privés. 


\section{Système membrane - couche d'air mince chez transducteurs électrostatiques et piézoplastiques}

\section{Z. ŠKVOR}

Faculté Electrotechnique, Université Technique Tchèque de Prague, Technická, 16627 Praha 6, Czech Republic

System membrane - air gap of an electrostatic and piezopolymer transducer

This paper describes analog models of electromechanical transducers with a diaphragm and the thin air film based on the electrostatic and piezoelectric (with piezopolymer membrane) principles.

\section{Introduction}

Dans la famille des transducteurs basés sur un champ électrique, ce sont les transducteurs électrostatiques et piézoplastiques, chez lesquels le système membrane - couche d' air joue un rôle principal.

La description de ce type de transducteur est réalisée le plus souvent au moyen des schémas équivalents analogiques facilitant une synthèse et $l^{\prime}$ optimisation.

Les traveaux les plus récents décrivent un système membrane - couche d' air, en considérant le milieu gazeux comme un fluide visco - thermique réel (M. Bruneau et al.) [1], [2].

Un transducteur électrostatique avec une membrane mince spécialement adapté pour la mesure de la pression acoustique dans une large bande de fréquences a été décrit par E. C. Wente (1917), [3]. Un an après, I. B. Crandall (1918) [4] a décrit un système de transducteur électrostatique dont la membrane est amortie par les pertes dûe à $l^{\prime}$ écoulement visqueux à $l^{\prime}$ entrefer.

Pour faire $1^{\prime}$ analyse d' un transducteur électrostatique, il est très convenient de décrire les deux parties du systême membrane - entrefer comme deux soussystèmes indépendants. Grâce a cette approximation on peut obtenir un modèle simple possédant une précision suffisante dans la gamme des fréquences fondamentales.

P. M. Morse (1948) [5] a défini la raideur quasistatique de la membrane circulaire (in vacuo) comme un facteur de proportionalité entre la force appliquée à la membrane et le déplacement moyen $\langle\xi\rangle$

$$
\begin{aligned}
s & =\frac{F}{<\xi\rangle}, \\
\langle\xi\rangle & =\frac{1}{S} \iint \xi d S .
\end{aligned}
$$


J. Merhaut (1967) [6] a déterminé un circuit équivalent de la membrane circulaire $d^{\prime}$ un transducteur électrostatique en introduisant le déplacement volumique de la membrane

$$
\Xi=\iint \xi d S
$$

et en définissant une impédance acoustique de la membrane comme le rapport du phaseur de la pression acoustique incidente sur celui du débit volumique $w=j \omega \Xi$

$$
\mathbf{Z}_{\mathbf{a}}=\frac{\mathbf{p}}{j \omega \Xi}=\frac{\mathbf{p}}{j \omega \iint \xi d S}
$$

Z. Škvor (1967, 1971) [7], [8] a calculé la résistance de l' entrefer d' un trasducteur électrostatique avec une électrode fixe perforée. La répartition de $l^{\prime}$ écoulement a été étudiée sur un modele hydrodynamique. Une suspension aqueuse $d^{\prime}$ un colorant en poudre mise entre deux plaques rigides dont une représentait une membrane et $1^{\prime}$ autre une électrode fixe, formait une couche mince simulant un milieux fluide incompressible. En écartant les plaques suivant une direction normale, les particules de colorant se déplaceaient en formant des figures qui représentaient les frontières entre domaines d' écoulement contigüs.

De ce modèle, en retenant la loi de Poiseuille a été calculé $1^{\prime}$ amortissement de la membrane en prenant en compte $l^{\prime}$ influence de la résistance acoustique de $l^{\prime}$ entrefer.

Plusieurs auteurs ont étudié $1^{\prime}$ impédance de $\mathrm{l}^{\prime}$ entrefer et du système membrane - entrefer: D. H. Robey (1954) [9], I. G. Petritskaya (1966) [10], J. Richter (1969) [11], J. E. Warren et al. (1973) [12], A. J. Zuckerwar (1978) [13], W. Kainz (1993) [14].

\section{Le circuit équivalent du système du microphone électrostatique}

Le circuit équivalent du microphone électrostatique, dont les parties fondamentales forment la membrane et l' entrefer, est très souvent représenté comme un trois- porte.

Une porte est électrique et les deux suivantes sont acoustiques. La surface externe de la membrane, sur laquelle agit la pression acoustique incidente $\mathbf{p}_{i}$, forme $1^{\prime}$ entrée de cet hexapôle, la surface interne chargée par l'entrefer forme la troisième porte.

Le but de tous les travaux théoriques est de créer un modèle analogique du transducteur. Les équations du problème sont celles qui régissent la vibration de la membrane et $I^{\prime}$ oscillation du fluide de $\mathrm{l}^{\prime}$ entrefer. Du point de vue de la modélisation, les deux parties, membrane et entrefer, sont des systèmes aux élements répartis et la déscription est limitée par une certaine gamme de fréquences, habituellement jusqu' au premier mode de la membrane.

Les travaux récents (M. Bruneau et al.) [1], [2] décrivent $1^{\prime}$ entrefer comme une couche fluide viscothermique à partir des équations de Navier - Stokes, de la conservation de la masse et de Fourier pour la conduction de la chaleur sous leurs formes linéarisées.

En négligeant les pertes dûes à la conduction de la chaleur on peut déduire un modèle classique du transducteur.

La membrane excitée sur la surface externe par une pression acoustique incidente $\mathbf{p}_{i}$ et sur la surface interne chargée par 1’entrefer, possède une impédance mécanique 


$$
\mathbf{Z}_{M}^{i e}=\frac{\mathbf{F}_{i}-\mathbf{F}_{e}}{\frac{j \omega}{S} \iint \xi d S}=\frac{\mathbf{p} S}{<\mathbf{v}>}=\frac{\mathbf{p} S^{2}}{\mathbf{w}}=\frac{\left(\mathbf{p}_{i}-\mathbf{p}_{e}\right) S^{2}}{<\mathbf{v}>S}
$$

ou $S$ est la surface de la membrane,

$\xi \quad$ le déplacement de la membrane,

$<\mathbf{v}>\quad$ la vitesse moyenne,

$\mathbf{w}=<\mathbf{v}>S \quad$ le débit volumique,

$\mathbf{F}_{\mathrm{i}}=\mathbf{p}_{\mathrm{i}} / S \quad$ la force dûe à la pression acoustique incidente,

$\mathbf{F}_{\mathrm{e}}=\mathbf{p}_{\mathrm{e}} / S \quad$ la force dûe à la charge acoustique de $\mathrm{l}^{\prime}$ entrefer effectuant sur la membrane.

$\mathrm{L}^{\prime}$ impédance acoustique de la couche $\mathrm{d}^{\prime}$ air de $\mathrm{l}^{\prime}$ enterfer est

$$
\mathbf{Z}_{E a}=\frac{\mathbf{p}_{e}}{\mathbf{w}}
$$

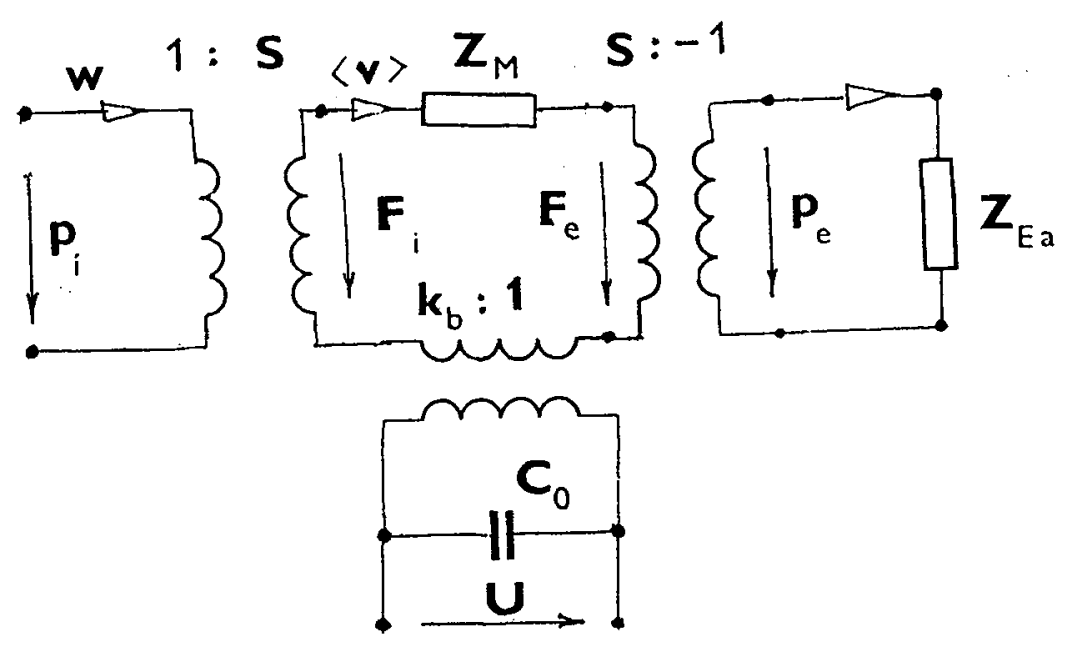

Fig. 1

La Fig. 1 décrit un schéma équivalent du transducteur électrostatique dont la troisième porte est représentée par les bornes électriques avec la capacitance $C_{0}$, la tension électrique de sortie $U$ et le rapport du transducteur

$$
k_{b}=\frac{Q_{0}}{h}
$$

$Q_{0}$ étant la charge électrique de polarisation et $h \mathrm{I}^{\prime}$ epaisseur de $\mathrm{I}^{\prime}$ entrefer.

$\mathrm{L}^{\prime}$ impédance acoustique de la memrane circulaire (in vacuo) (i.e. [8], [15]) est comme suit 


$$
\mathbf{Z}_{M a}=\frac{\mathbf{Z}_{M}}{S^{2}}=\frac{k_{M}^{2} v}{j \omega} \frac{1}{S} \frac{J_{0}\left(k_{M} R\right)}{J_{2}\left(k_{M} R\right)}
$$

où $\quad k_{\mathrm{M}} \quad$ est le nombre $\mathrm{d}^{\prime}$ onde,

$$
k_{M}=\omega \sqrt{\frac{m_{1}}{v}}=\frac{\omega}{c_{m}},
$$

$m_{1} \quad$ la masse surfacique,

$v \quad$ la tension de la membrane,

$c_{\mathrm{m}} \quad$ la vitesse $\mathrm{d}^{\prime}$ ondes sur la membrane,

$R \quad$ le rayon de la membrane.

$L^{\prime}$ impédance mécanique du système membrane - entrefer est

$$
\mathbf{Z}_{M E}^{*}=\mathbf{Z}_{M}+\mathbf{Z}_{E a} S^{2}+\frac{k_{b}^{2}}{j \omega C_{0}}
$$

ou, en négligeant le troisième terme

$$
\mathbf{z}_{M E}^{*} \approx \mathbf{z}_{M E}=\mathbf{z}_{M}+\mathbf{z}_{E a} S^{2}
$$

La sensibilité du microphone est

$$
\eta=\frac{\mathbf{U}}{\mathbf{p}_{i}}=\frac{S k_{b}}{k_{b}^{2}+j \omega C_{0} \mathbf{Z}_{M E}}
$$

et pour

$$
\omega C_{0} \mathbf{z}_{M E}>k_{b}^{2}
$$

on a

$$
\eta \approx \frac{k_{b} S}{C_{0}} \frac{1}{j \omega \mathbf{Z}_{M E}}
$$

En supposant le déplacement moyen de la membrane on peut déduire les élements du système membrane - entrefer présentés sur la Fig.2 pour la gamme des fréquences fondamentales:

$$
\mathbf{Z}_{M} \approx m_{M}+\frac{1}{j \omega c_{M}}
$$

est $l^{\prime}$ impédance mécanique de la membrane, où

$m_{\mathrm{M}} \quad$ représente $\mathrm{l}^{\prime}$ inertance de la membrane circulaire

$$
m_{M}=1,4458 m_{1} \pi R^{2} \quad[15],
$$

$c_{\mathrm{M}} \quad$ la compliance mécanique de la membrane

$$
c_{M}=\frac{1}{8,3612 \pi v}
$$

$c_{\mathrm{E} 1} \quad$ la compliance mécanique de $\mathrm{l}^{\wedge}$ entrefer

$$
c_{E 1}=\frac{h}{\rho c^{2} \pi R^{2}},
$$




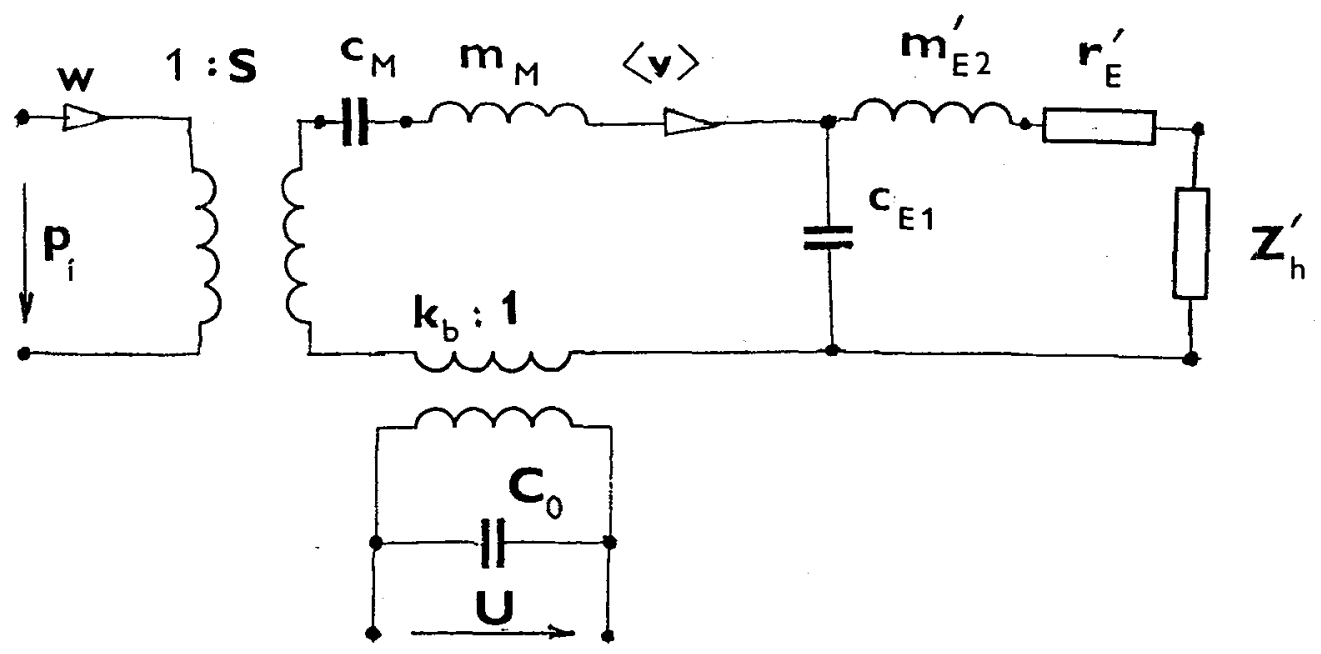

Fig.2

$r_{E}^{\prime} \quad$ la résistance mécanique de $\mathrm{l}^{\prime}$ entrefer dûe aux pertes visqueuses

$$
r_{E}^{\prime}=3 \mu \frac{\pi R^{4}}{h^{3}}
$$

$m_{\mathrm{E} 2}^{\prime} \quad l^{\prime}$ inertance de l' entrefer

$$
m_{E 2}^{\prime}=\frac{1}{2} \rho \frac{\pi R^{4}}{h}
$$

$\mathbf{Z}_{\mathrm{h}}^{\prime} \quad \mathrm{I}^{\prime}$ impédance mécanique du réservoire périphérique transformée sur la membrane vibrant avec une vitesse moyenne $<v>$.

La résistance $r_{\mathrm{E}}^{\prime}$ calculée à partir de la loi de Poiseuille [7] pour le mode en piston est

$$
r_{E}=\frac{3 \mu}{2} \frac{\pi R^{4}}{h^{3}}=\frac{\mathbf{F}_{r}}{v_{0}}
$$

ou $F_{r}$ est la force d' amortissement éffectuant sur la membrane, et $v_{0}$ la vitesse uniformément répartie sur la membrane.

En supposant la vitesse moyenne (en cas de déplacement parabolique) $\left\langle v>=v_{0} / 2\right.$, on obtient

$$
r_{E}^{\prime}=\frac{3 \mu \pi R^{4}}{h^{3}}
$$

Cette valeur est en bon accord avec le terme

$$
r=\frac{11}{4} \mu \frac{\pi R^{4}}{h^{3}}
$$

derivé de [4]. 
Le schéma, ainsi que les valeurs des élements de la Fig.2 sont très voisines de celles calculées en [2] pour la gamme des fréquences fondamentales.

Pour la plupart des applications, la description du transducteur présentée ici a une precision suffisante.

Cependant, pour des applications plus pointues il est possible d’ affiner la description.

\section{Transducteur piézoplastique et son circuit équivalent}

Le tranducteur piézoplastique est de la famille des transducteurs, qui sont très proches de ceux basés sur le principe electrostatique.

Sur la Fig.3 nous présentons comme example un transducteur piézoplastique de forme spiral [16],[17], [18], [19] fonctionant comme un écouteur.
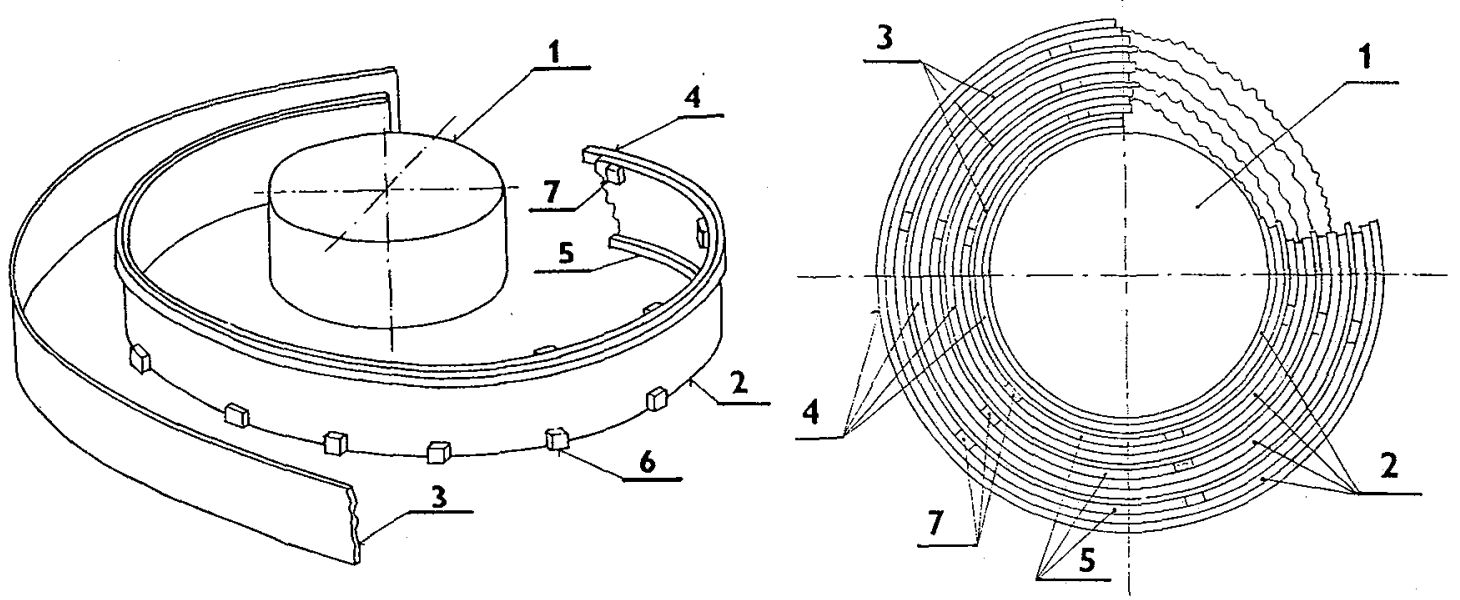

Fig.3

Sur le noyau 1 est enroulée une bande piézoplastique 2 avec les pièces $4,5,6,7$ et une bande séparatrice 3. Les bandes de forme cylindrique 2 et 3 forment entre elles des entrefers. La bande piézoplastique en changeant sa longueur sous 1' influence du champ électrique d' excitation, vibre dans la direction radialë. étant chargée par les couches minces $d^{\prime}$ air entre les bandes 2 et 3 . Ce système possède le schéma équivalent dessiné sur la Fig.4, ou

$\mathrm{U}_{3} \quad$ est la tension électrique $\mathrm{d}^{\prime}$ excitation ,

$c_{\mathrm{N}}$ la compliance de la bande,

$m_{\mathrm{N}}$ la masse de la bande,

$C_{e 0 N}=\varepsilon_{33}^{T} N \frac{1-k_{31}^{2}}{h} 2 \pi R b \quad$ la capacitance de tranducteur,

$N \quad$ le nombre de tours de la bande,

$h \quad$ l'épaisseur de la bande piézoplastique, 
$\varepsilon_{33}^{T}$ la permitivité du matériel piézoplastique,

$k_{31} \quad$ le coefficient de couplage électromécanique,

$R \quad$ le rayon moyen de la bande,

$b$ la largeur de la bande,

$\mathbf{Z}_{2}=j \rho_{0} c_{0} N 2 \pi R H\left(-\cot k b+\frac{1}{k b}\right) \quad l^{\prime}$ impédance de $1^{*}$ entrefer,

$k \quad$ le nombre d'onde,

$H \quad 1^{\prime}$ épasisseur de $1^{\prime}$ entrefer,

$R_{m}=8 \mu \pi R b \frac{N}{H}$ la résistance de $1^{\prime}$ entrefer,

$k_{b N}=N 2 \pi b \frac{d_{31}}{s_{11}^{E}}$ le coefficient du transducteur,

$c_{m}=\frac{H}{\rho_{0} c_{0}^{2} N b 2 \pi R} \quad$ la compliance de $l^{\prime}$ entrefer.

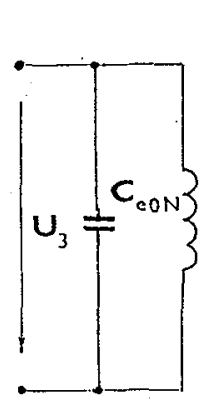

1: $k_{b N}$
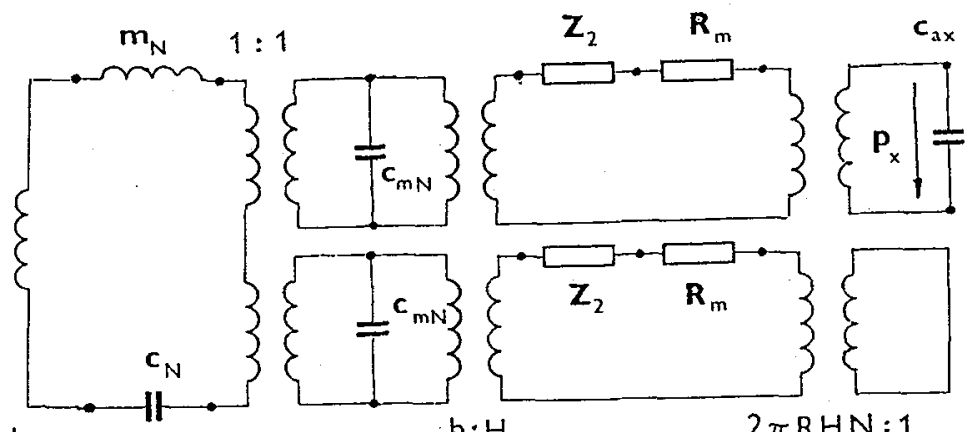

$b: \mathrm{H}$

$2 \pi$ RHN $: 1$

Fig.4

La réponse $\mathrm{d}^{\prime}$ un écouteur à la bande piézoplastique chargé $\mathrm{d}^{\top}$ un côté par une cavité de volume $1,4 \mathrm{~cm}^{3}$ ( $1^{\prime}$ autre côté étant sans charge acoustique), est représentée, pour la tension $U_{3}=5 \mathrm{~V}_{5}$ sur la Fig.5.

Les courbes de rẻponses $(\mathrm{R}=5 \mathrm{~mm}, \mathrm{~N}=5, \mathrm{H}=60 \mu \mathrm{m}, \mathrm{b}=15 \mathrm{~mm}, \mathrm{~h}=25 \mu \mathrm{m}$ - film piézoplastique PVDF - Solef) théorique. et mesurée sont en bon accord.

\section{FAEQ RESP}

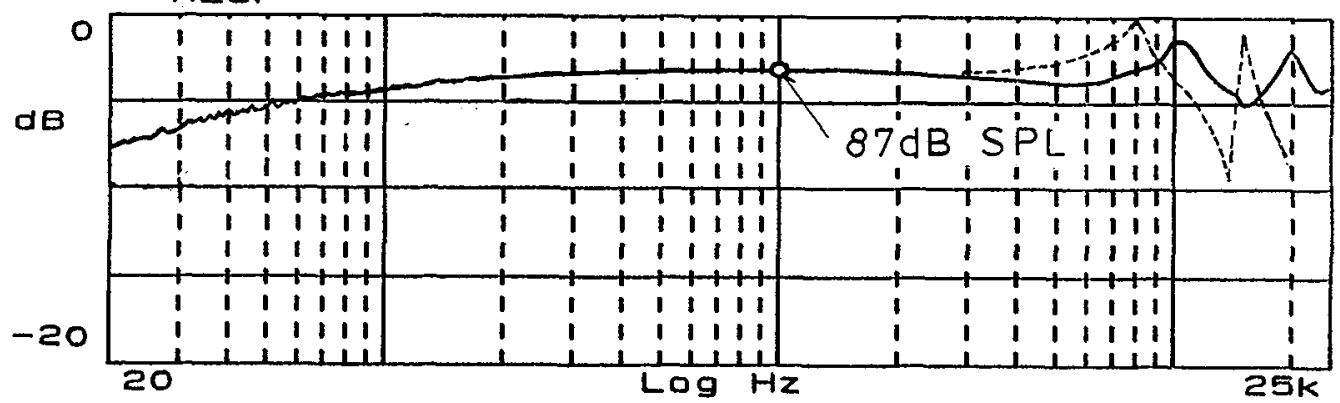

Fig.5 


\section{Resumé}

Nous avons présenté deux types de modèles de transducteurs basés sur la théorie simplifiée facilitant une étude synthétique et analytique efficace, étant très utile pour la réalisation des transducteurs.

\section{Bibliographie}

[1] Bruneau, M., et al.: Modelisation des microphones miniatures: Effets des couches limites visco-thermique. J. Ph. IV, Coll.C1, Vol.2, Avril 1992, $2^{\text {Łme }}$ Conf. Fr. d'Acoust., Arcachon

[2] Bruneau, M. et al.: An electrical network to modelling the strong coupling between s vibrating membrane and a fluid film. To be published in Acta Acustica

[3] Wente, E. C.: Phys. Review, Vol.X, Series II, 1917, No.1, pp.39 - 63

[4] Crandall, I. B.: Phys. Review, Vol.XI, Series II, 1918, No.6, pp.449 - 460

[5] Morse, P. M.: Vibration and Sound, Sec. ed., Mc Graw- Hill , N.Y., 1948, pp.195 - 203

[6] Merhaut, J.: Acustica, 19, No.5, 1967/68, pp. 284 - 292

[7] Škvor, Z:: Acustica, 19, No.5, 1967/68, pp. 295 - 299

[8] Škvor, Z.: Hochfrequenz und Elektroakustik, 80, No.3, Leipzig 1971, pp. 85 - 92

[9] Robey, D. H.: J. Acoust. Soc. Am., 26, 1954, pp. $740-745$

[10] Petritskaya, I. G.: Sov. Phys. Acoust., 12 (2) , 1966, pp. 193 - 198

[11] Richter, J.: Acustica 22, No.2, 1969/70, pp. 95 - 106

[12] Warren, J. E. et al. :J. Acoust. Soc.Am., 54 (5), 1973, pp.1201 - 1213

[13] Zuckerwar, A. J.: J. Acoust. Soc. Am., 64 (5), 1978, pp. $1278-1285$

[14] Kainz, W.: Coupled Acoustic Systems, Fortschritte VDI, Reihe 11, Nr.186, Düsseldorf, 1993

[15] Škvor, Z.: Vibrating Systems and their Equivalent Circuits. Elsevier Sc. Publ., Amsterodam,1991

[16] CZ Pat. Appl, 277814, 277815

[17] Škvor, Z.: Transducteurs électroacoustiques et électromécaniques et leurs circuits équivalents, J. Phys. Vol.2, Coll.No.1, Suppl.JP III, No.4, $2^{\text {zme }}$ Congr. Fr. Acoust.Vol, 1, C1-349-356

[18] Škvor, Z. et al.: Acta Polytechnica, CTU, Vol .32, No.1/1992, pp.59 - 64

[19] Lotton, P.: Rapport de stage D. E. A., Acoust. Appl., CTU Prague, LAUM, Le Mans, 1991 